\title{
Raman Study of the Intermolecular Interactions between Thermotropic Liquid-Crystalline Oligomers: Comparison of the Effect of Poly(Propylene Oxide) and Poly(Ethylene Oxide)
}

\author{
Keunok Han Yu, ${ }^{\dagger}$ John Moon Rhee, ${ }^{\dagger}$ Jung-Hoon Lim, and Soo-Chang Yu ${ }^{*}$ \\ ${ }^{\dagger}$ Polvmer Science and Technologv, Chonbuk Rational University. Chonju. Chonbuk $561-756$, Korea \\ Deparment of Chemistry, Kunsan National Unversity. Kunsan, Chonbuk 573-701, Korea \\ Received November 27, 2001
}

Keywords : Raman, Liquid crystal. Intermolecular interaction, Coil effect, Hydrogen bonding.

\section{Introduction}

Liquid crystalline polymers (LCPs) have been intensively studied for their scientific and technological interests. Among LCPs, rod-coil types have recently drawn attention, particularly in relation to their likelihood to form various kinds of supramolecules depending on chain lengths. The LCPs based on a biphenyl ester moiety can have different microscopic structures. Most poly (ethylene oxide)s (PEO)-based LCPs result in a layered smectic mesophase, ${ }^{]}$whereas poly(propylene oxide)s (PPO)-based LCPs give rise to a layered smectic C. smectic A. biconticuous cubic, and hexagonal columnar mesophases depending on the chain lengths." Considering the fact that the physical properties of LCPs are mainly characterized by the molecular interactions between the mesogenic moieties, the molecular-based information about the coil effects on the molecular interactions between the mesogenic moieties is very important in providing an insight into the mechanism for the formation of supramolecules. $^{3}$

Enormous efforts have been undertaken to investigate the intermolecular structure of LCPs using the fluorescence technique. ${ }^{4}$ Although it is highly sensitive to the intermolecular structure as to detect only small differences in the chain lengths, this method is limited in yielding information regarding the specific sites involved in the intermolecular interactions. Vibrational spectroscopy such as infrared or Raman is considered among the most efficient tools for this purpose. providing rich information on a molecular level. For morphological studies. Raman spectroscopy is recognized to be superior to infrared spectroscopy because a modification of the samples is not incurred during Raman measurement, which is critical to the preservation of morphology.

In order to study the coil effects on the intermolecular structure by employing Raman spectroscopy, we have chosen a rod-coil liquid crystalline oligomer with twelve PPO units as a coil (PI2-4) and compared its results with those of $12-4$ with twelve PEO units. the esterification products of ethyl 4 [4'-oxy-4-biphenylcarbony loxy]-4'-biphenylcarboxylate with PPO (DP=12) and with PEO (DP=12). To this end three

Comesponding author. E-mail: scy uakunsan ac.kr: Fax: +82$63-466-2085$ different vibrational modes(carbonyl, aromatic $\mathrm{C} \cdot \mathrm{H}$ and aromatic $\mathrm{C}=\mathrm{C}$ ) were investigated at various temperatures, which may unambiguously provide complementary information about the intermolecular structure in the crystalline phase.

\section{Experimental Section}

The details of the synthesis and characterization of P12-4 and 12-4 were reported in a previous publication. ${ }^{1.2}$ The crystalline to LC phase transition temperatures (first heating at a rate of $10^{\circ} \mathrm{C} \mathrm{min}^{-1}$ ) were observed at $36.9^{\circ} \mathrm{C}$ and 135.2 ${ }^{\circ} \mathrm{C}$, for P12-4 and 12-4, respectively, from a Perkin Elmer DSC-7 differential scanning calorimeter (DSC) equipped with a 1020 thermal analysis controller.

The Raman spectra were obtained using a Renishaw Raman micro system 2000 with the 632.8-nm line of a HeNe laser as excitation source. The laser power at the sample was ca. $5 \mathrm{~mW}$ and the resolution of the spectra was within \pm 2 $\mathrm{cm}^{-1}$. Putting the samples on glass slides, the data were acquired by using a Linkam heating and freezing stage (THMSE 600) with a controller (TMS 92). and then calibrated to the silicon band at $52 \mathrm{~cm}^{-1}$.

\section{Results and Discussion}

We first considered carbonyl modes since their modification directly indicates the existence of intermolecular interactions such as hydrogen bonding or dipole-dipole interaction. Nomally there are only two carbonyl bands in the spectra of both P12-4 and 12-4 unless intermolecular interactions exist. The two carbonyl groups in each molecule are surrounded by different environments. retaining a different force constant around the $\mathrm{C}=\mathrm{O}$ bond. In fact. only two apparent bands were observed in our previous report at 1718 and $174 \mathrm{~cm}^{-1}$ when $-\mathrm{CH}_{2} \mathrm{CH}_{3}$ of $12-4$ was replaced by $-\left(\mathrm{CH}_{2}\right)_{21} \mathrm{CH}_{3}$ to interfere with the intermolecular interactions. ${ }^{6}$ This was proven to be true for PI2-4 in our recent experiment (not shown here). Of the two bands. the low wavenumber band corresponds to the $\mathrm{C}=\mathrm{O}$ group between the biphenyl moieties, while the high wavenumber band corresponds to the $\mathrm{C}=\mathrm{O}$ group near the ethyl terminal. ${ }^{7}$ For both P12-4 and 12-4, however. three bands, 1709. 1715, 


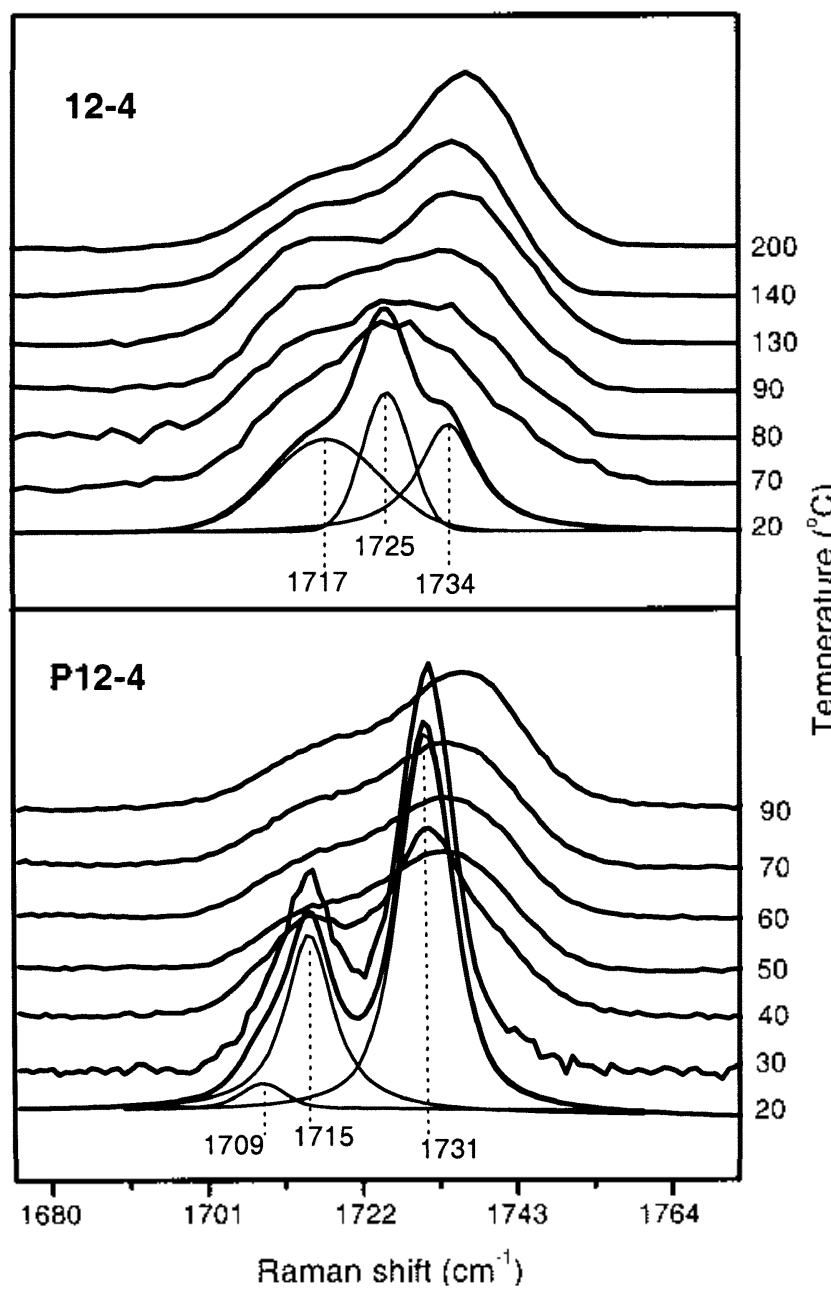

Jigure I, lemperature dependence of the carbonyl modes for P124 (bottom) and $12-4$ (top). The three solid lines are obtained through curve-fitting of the corresponding spectrum at 20 " $\mathrm{C}$.

$1731 \mathrm{~cm}^{-1}$ for P12-4, and $1717,1725,1734 \mathrm{~cm}^{-1}$ for $12-4$ were observed at low temperatures as can be seen in Figure $\mathrm{l}$, indicating the existence of intermolecular interactions in the crystalline phases. The band at $1709 \mathrm{~cm}^{-1}$ seems not to be prominent here, but it was still existed in a series of molecules with different length of $\mathrm{PPO}$ coil.

The locations of the two free carbonyl bands for PI2-4 should be nearly the same as those of 12-4 since the two molecules have the same mesogenic units. Therefore, the bands at $1715 \mathrm{~cm}^{-1}$ and $1717 \mathrm{~cm}^{-1}$ for $\mathrm{P} 12-4$ and for $12-4$, respectively, are assignable to the $\mathrm{C}=\mathrm{O}$ groups between the biphenyl moieties, whereas the bands at $1731 \mathrm{~cm}^{-1}$ and 1734 $\mathrm{cm}^{-1}$ for $\mathrm{P}^{\mathrm{P}} 12-4$ and for $12-4$, respectively, to the $\mathrm{C}=\mathrm{O}$ groups near the ethyl terminal. Accordingly, the bands at $1709 \mathrm{~cm}^{-1}$ and $1725 \mathrm{~cm}^{-1}$ for P12-4 and $12-4$, respectively, can be assigned to the hydrogen bonded carbonyl modes. Considering the fact that the $\mathrm{C}=\mathrm{O}$ band shifts to lower wavenumbers upon hydrogen bonding. ${ }^{8}$ the band at $1709 \mathrm{~cm}^{-1}$ originates from the $\mathrm{C}=\mathrm{O}$ group between the biphenyl moieties, whereas the band at $1725 \mathrm{~cm}^{-1}$ originates from the $\mathrm{C}=\mathrm{O}$ group near the ethyl terminal. One may argue that this might be due to one of the following phenomena: dipole-dipole inter-

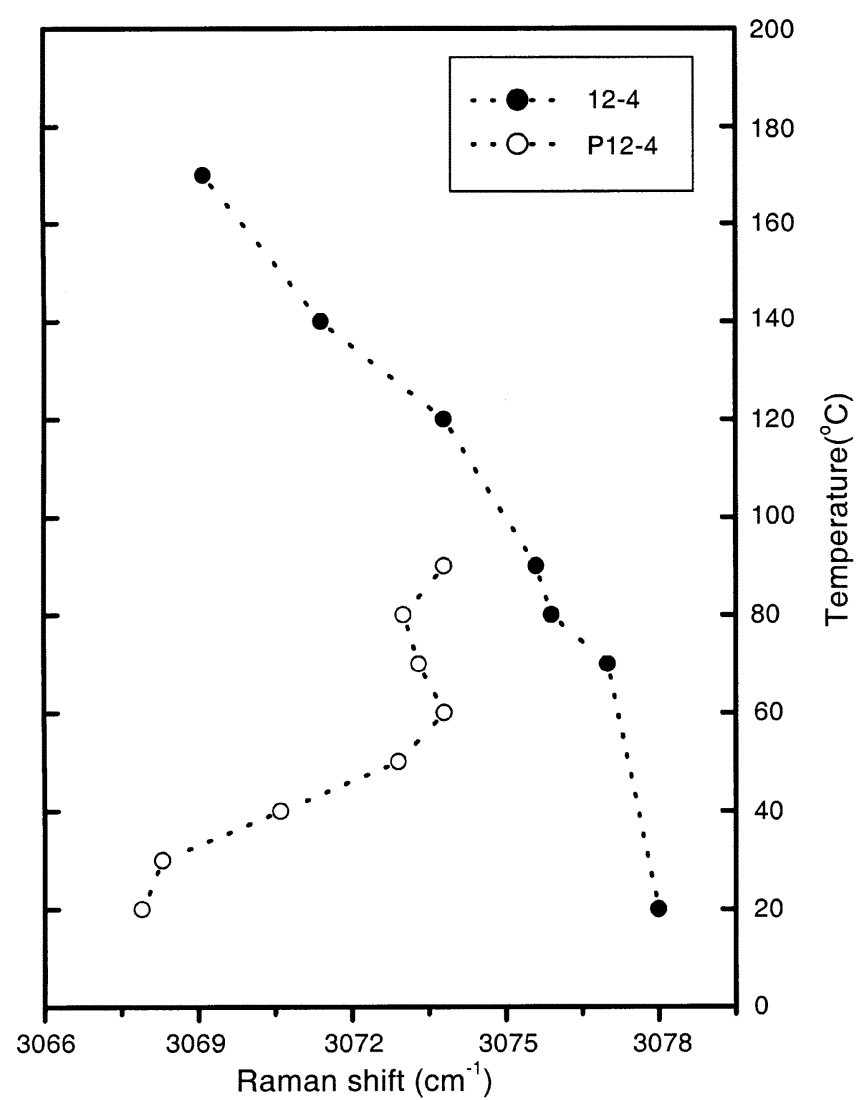

Figure 2, lemperature dependence of the C-H suretching modes for P12-4 ( a) and $12-4(\bullet)$

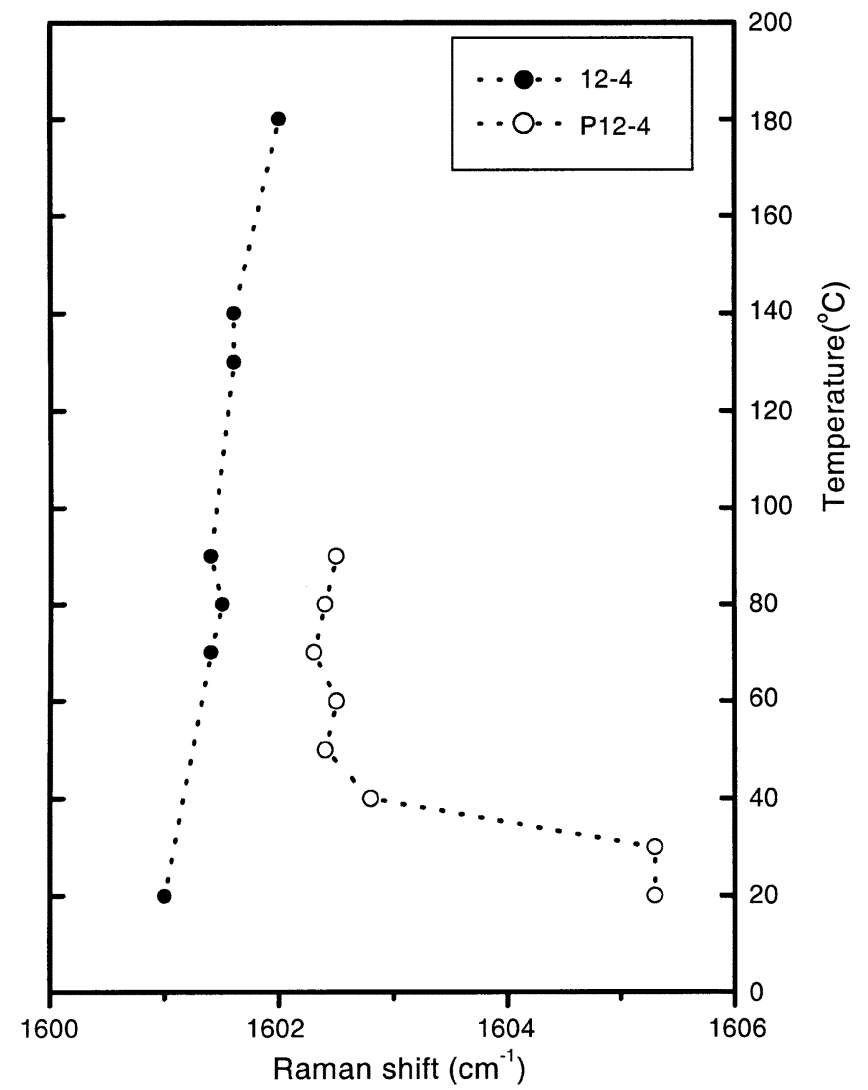

Figure 3. l'mperature dependence of the $\mathrm{C}=\mathrm{C}$ stretching modes tor P12-4 ( $\ldots)$ and $2-4$ 
$12-4$

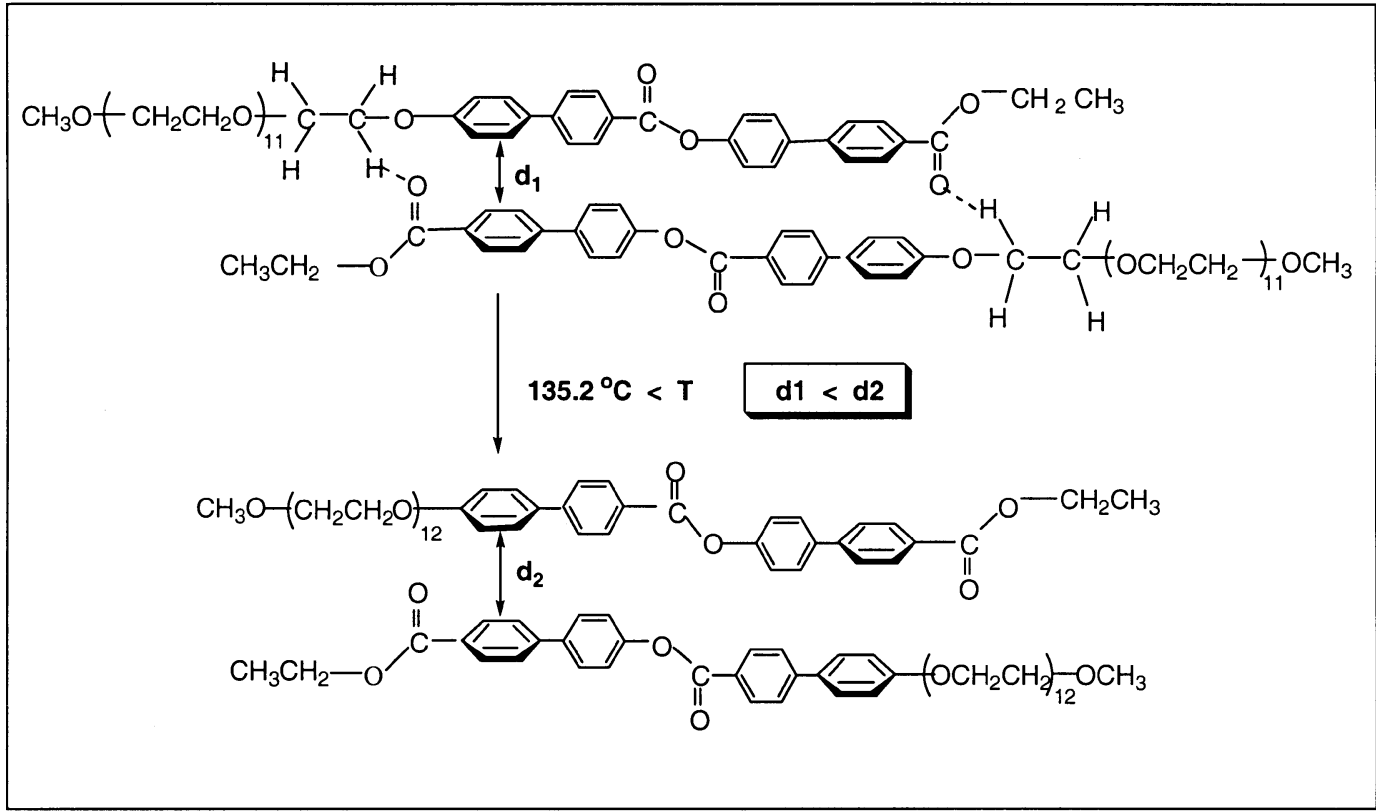

\section{P12 - 4}

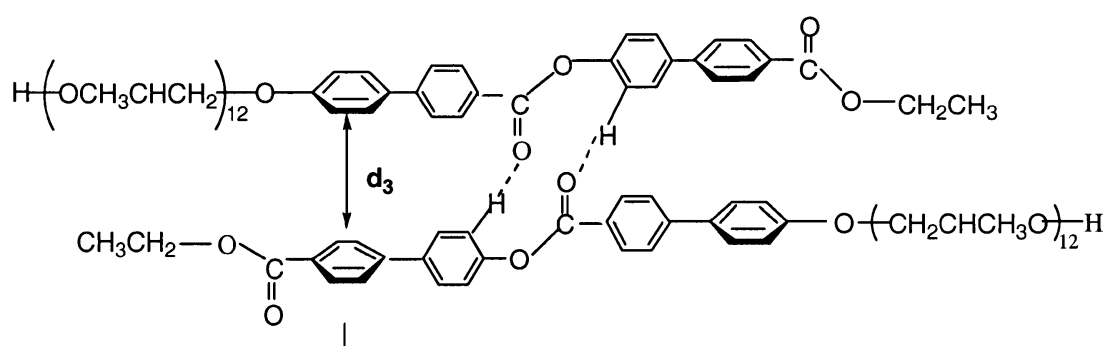

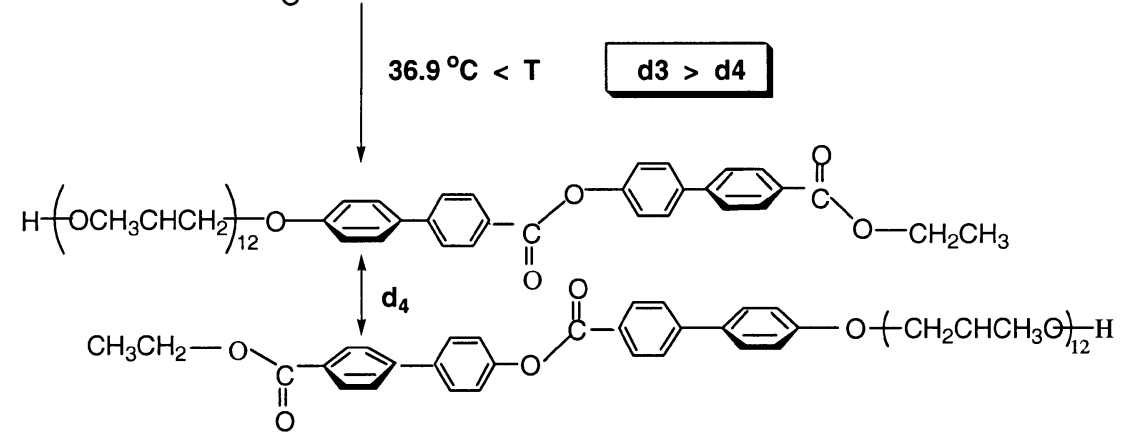

Figure 4. Schematic diagram for the intermolecular arrangements of PI2-4 and 12-4 before and after the phase transition temperatures. The distance hetween neighboring mesogens is designated by $d_{i}(i=1.2 .3 .4)$.

action. conformers, or hydrogen bonding with $\mathrm{H}_{2} \mathrm{O}$ from moisture. However, we ruled out dipole-dipole interaction because we hardly explain the reason why the aromatic $\mathrm{C}-\mathrm{H}$ band for each molecule shifted towards an opposite direction. The possibility of conformers was already investigated in our previous experiment, ${ }^{7}$ and it was excluded here. The hydrogen bonding due to the moisture was also carefully considered, and ruled out because it is not reasonable for only one carbonyl group to be involved in hydrogen bonding. Moreover, no corresponding band was observed when $\mathrm{CH}_{2} \mathrm{CH}_{3}$ was replaced with $\left(\mathrm{CH}_{2}\right)_{2} \mathrm{CH}_{3}$ for both $12-4$ and P12-4.

It is noteworthy that the $\mathrm{C}=\mathrm{O}$ band at higher frequency shifts abruptly around the phase transition temperature for P12-4 (compare the spectrum at $40{ }^{\circ} \mathrm{C}$ with that at 50 )." whereas that of 12-4 shifts gradually over the entire temper- 
ature range. This seems to be mainly due to the different disruption mechanism for intermolecular interactions during phase transitions. According to our previous results, the carbonyl group near the ethyl terminal was easier to rotate than the one between the biphenyl groups during the phase transition. ${ }^{7}$ Therefore. the bond rotation about the aromatic $\mathrm{C} \cdot(\mathrm{C}=\mathrm{O})$ is gradually followed by bond breaking. resulting in the continuous frequency shift owing to the conformational change for $12-4$. On the other hand, as the hydrogen bonding is broken down, only the distance between the neighboring mesogens changed, without giving rise to bond rotation about the aromatic $\mathrm{C} \cdot(\mathrm{C}=\mathrm{O})$ (between the biphenyl moiety) participating in the hydrogen bonding for P12-4. Accordingly, the frequency shift is not developed above the phase transition temperature $\left(50^{\circ} \mathrm{C}\right)$

The above argument can be clarified by the counterpart of the $\mathrm{C}=\mathrm{O}$ group involved in the hydrogen bonding. This is supported by the observation of the temperature dependent behavior of the aromatic $\mathrm{C} \cdot \mathrm{H}$ stretching modes. Assuming that one of the aromatic hydrogen atoms is responsible for the hydrogen bonding. the aromatic $\mathrm{C}-\mathrm{H}$ stretching mode is. then, anticipated to shift to a higher frequency upon breaking down the hydrogen bonding since the weakened $\mathrm{C} \cdot \mathrm{H}$ bond will be reinforced. The result for $\mathrm{P} 12-4$ showed that the frequency shift to higher wavenumbers from $3068 \mathrm{~cm}^{-1}$ in the crystalline phase to $3074 \mathrm{~cm}^{-1}$ in the molten phase is consistent with our assumption (Figure 2). In fact. the type of $\mathrm{C}-\mathrm{H} \cdots \mathrm{O}$ hydrogen bonding has been revealed by theoretical and experimental studies. ${ }^{1011}$ The result for $12-4$. however. shows the reverse direction to lower wavernumber. ruling out the hydrogen bonding with the aromatic hydrogen atomis. The downwward frequency shift of this aromatic $\mathrm{C} \cdot \mathrm{H}$ stretching band from 3078 to $3069 \mathrm{~cm}^{-1}$ is found to be due to expansion of the packing structure. ${ }^{12}$ The possibility of the hydrogen bonding of the $\mathrm{C}=\mathrm{O}$ group with one of the hydrogen atoms of the PEO group for 12-4 was suggested in our previous report, ${ }^{7}$ and is confinned through the observations described above.

This can be further confirmed by observing the $\mathrm{C}=\mathrm{C}$ stretching mode. The aromatic $\mathrm{C}=\mathrm{C}$ band displays a downward shift in frequency for P12-4. whereas it displays an upward shift for $12-4$ (Figure 3). Considering the fact that a downward shift in frequency of the $\mathrm{C}=\mathrm{C}$ band is generally due to the decrease in the inter-chain force constant. ${ }^{8}$ the downward shift for P12-4 seems to be due to the decrease in the force constant about the $\mathrm{C}=\mathrm{C}$ bond probably resulting from the conjugation between the neighboring mesogens. In the crystalline phase. hydrogen bonding is formed through the carbonyl bond between the biphenyl moieties with the adjacent aromatic hydrogen atom. maintaining a certain distance between neighboring mesogens. As the hydrogen bonding is broken down at the phase transition temperature. this distance becomes closer. resulting in a $\pi-\pi$ stacking. Thus the $\pi-\pi$ stacking causes the frequency shift to lower wavenumber since the $\pi$ electrons of the benzene ring participating in the $\pi-\pi$ stacking reduce the force constant of the $\mathrm{C}=\mathrm{C}$ bond. The upward shift in 12-4 can be explained by a quite opposite interpretation. It seems that the $\pi-\pi$ stacking formed between the biphenyl groups below the phase transition temperature becomes loosened upon reaching the phase transition temperature giving rise to the upward slift. As is the case for the aromatic $\mathrm{C} \cdot \mathrm{H}$ stretching mode, the frequency shift occurs only during the phase transition for P12-4, while it occurs monotonously with increasing temperature for 12-4. The schematic diagram for the changes in the intermolecular interactions around the phase transition temperature can be drawn as shown in Figure 4.

\section{Conclusions}

The coil effects on the intermolecular structure in the crystalline phase were studied by observing the three Raman modes. $\mathrm{C}=\mathrm{O}$, aromatic $\mathrm{C} \cdot \mathrm{H}$. and aromatic $\mathrm{C}=\mathrm{C}$. $\mathrm{PPO}$ and $P E O$ coils are found to influence the intermolecular structures in a different way. The complementary results from the vibrational modes indicate that in PI2-4 with PPO coils, the hydrogen bonding is induced through the carbonyl group between the biphenyl groups with the aromatic hydrogen atom of the adjacent molecule. In contrast, in 12-4 with PEO coils. the hydrogen bonding is induced through the carbonyl group near the ethyl terminal with the hydrogen of PEO coil of the adjacent molecule. Regardless of whether or not the intermolecular interaction can be assigned to a hydrogen bonding, our conclusion that $\mathrm{PPO}$ and $\mathrm{PEO}$ coils affect the molecular arrangement in a different way is still valid. These different arrangements in the crystalline phases may play an important role in making different LC structures.

Acknowledgment. This work was supported by the grant of Post-Doc. Program. Chonbuk National University (1999).

\section{References}

1. Lee. M: Oh, N.-K. J. Hater Chem. 1996, 6, 1079

2. Lee M.: Cho. B.-K.: Kim. H.: Yoon. J.-Y: Zin. W-C. J. An Chent Soc. $1998.120,13258$.

3. Bahadur. B. Liquid Cristals: Applications and Lses: World Scientifie Publishing Co: Singapore, 1990.

4. Huang. $\mathrm{H}$. W: Horie, K.: Tokita, M.: Watanabe J. Macromol. Chem. Phys. 1998, 199. 1851

5. Kroschwitz. T. I. Polmers: Pohnter Characterization and Anahysis: John Wiley \& Sons: New York. 1990.

6. Yu. S.-C.: Yu. K. H.: Yu. T.: Lee. M. Bull. Korem Chent. Soc 1998. 19.885

7. Yu. S.-C:; Choi, Y: Yu, K. H; Yu, J.; Choi, H,; Kim, D. H:; Lee, M Macronlecules $\mathbf{2 0 0 0}$.33.6527.

8. Wu. P. P.: Hsu. S. L.: Thomas. O.: Blumstein. A. J. Polym. Sci. Part B Polnter Phsics 1986. 24.827.

9. Since the intensity of the hydrogen-bonded carbonyl mode is too low to monitor with temperature we monitored the free carbonyl mode at higher frequency. This is because its spectral behavior can be affected by the disruption of the conjugated form as the hydrogen bonding is broken down.

10. Hartmant1. M.: Wetmore. S. D.: Random. L. J. Phos Chem. A 2001. 105. 4470 .

11. Kariuki. B. M.: Harris. K. D. M.; Philip, D.; Robinson. J. M. A. J. Am. Chem. Soc. 1997, 119, 12679.

12. Briscoe. B. J.: Stuart. B. H.: Thomas. P. S.; Williams. D. R. Spectrochint deta 1991. +7.4. 1299. 